\title{
SYNTHESIS AND CHARACTERIZATION OF SOME NEW BIOLOGICALLY ACTIVE PYRIMIDINE DERIVATIVES
}

\author{
Uttam B. More \\ Postgraduate Department of Chemistry, Sadguru Gadage Maharaj College, Karad, \\ Pin 415124, (M.S.) India \\ E-mail: uttambmore@ rediffmail.com
}

\begin{abstract}
Pyrimidine Compounds have occupied an important position in natural and synthetic organic chemistry, mainly due to their wide range of biological activities. Here we have synthesized some new pyrimidine compounds using urea, ethyl cyanoacetate and different aldehydes, in moderate to good yields.
\end{abstract}

Keywords: Pyrimidine, multi-component reactions, biological activity.

(C) RASĀYAN. All rights reserved

\section{INTRODUCTION}

The heterocyclic aromatic systems are of much greater importance than the constituent monocyclic compounds. The appearance of qualitatively new properties of an annulated molecule, enlargement of the possibility of varying pharamacophore groups in different positions of the molecule and ability of the latter to interact with a wider spectrum of receptors adopting various conformations are apparently of crucial importance.

The aspect of uracil and its derivatives was well recognized by organic as well as biologists. With the development of clinically useful anticancer and antiviral drugs, there has recently been remarkable interest in the synthetic manipulation of uracils. Multi-Component reactions, in which multiple reactions are combined into one synthetic operation, have been used extensively to form carbon-carbon bonds in synthetic chemistry. Such reactions offer a wide range of possibilities for the efficient construction of highly complex molecules in single procedural steps, avoid the complicate purification operation and allow savings of both solvents and reagents. In the past decade, there has been a tremendous development in three and four component reactions and great efforts continue to be made to develop new multicomponent reactions. The need to reduce the amount of toxic waste and by-product arising from chemical processes requires increasing emphasis on the use of less toxic and environmentally compatible materials in the design of new synthetic methods.

In modern years, pyrimidine and dihydropyrimidines have been in an important position in natural and synthetic organic chemistry, due to their wide range of biological activities, such as antibacterial, antiviral, antihypertensive and antitumor effects and their effects as calcium channel blockers. ${ }^{1-13}$ The development of efficient and environmentally acceptable synthetic methods is an important task of modern chemistry. Conventional organic syntheses are generally based on homogeneous catalysts. However, homogeneous reactions suffer disadvantages in separation, isolation of products and so on. From the view point of green chemistry, the use of heterogeneous catalysts is desirable. The consequential advantages of heterogeneous catalysts from the environmental and economic points of view are clearly understandable, because these procedures are low costing and the production of the waste material is decreased. In contrast to the extensive studies involving heterogeneous acid catalysts, fewer efforts have been made to develop heterogeneous base catalysts. Several solid bases have been reported as being effective in this respect, such as zeolites, ${ }^{14}$ alkali metals supported on alumina, ${ }^{15}$ clay minerals, metal oxides such as magnesium oxide, ${ }^{16}$ and mixed metal oxides, for example, magnesium - lanthanum mixed oxide.

Rasayan J. Chem., 11(3), 1217-1221(2018)

http://dx.doi.org/10.31788/RJC.2018.1133062

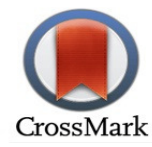


Among these solid bases, $\mathrm{MgO}$ recently has been studied using a novel but simple procedure, was systematically investigated as a heterogeneous base catalyst for reactions taking place in the liquid phase, specifically the Michael addition and the Knoevenagel condensation.

\section{EXPERIMENTAL}

The chemicals and solvents used for the experimental work were commercially procured from Sd. fine Chemicals. The melting points of all synthesized compounds were determined by open tube capillary in the Celsius scale and uncorrected. IR spectra were recorded using $\mathrm{KBr}$ pellets on Perkin Elmer spectrophotometer, ${ }^{1} \mathrm{H}$ - NMR spectra of the final compounds were recorded on Bruker NMR spectrometer $(300 \mathrm{MHz})$.

\section{General Procedure for Synthesis of Compounds 1(a-i)}

A mixture of ethyl cyanoacetate $(10 \mathrm{mmol})$, thiourea $(10 \mathrm{mmol})$ and appropriate aldehydes $(10 \mathrm{mmol})$ was stirred in a solution of sodium acetate for 48 to $54 \mathrm{hr}$ and then the reaction mixture was poured in crushed ice. Then it was acidified and the product obtained was filtered off, then dried and recrystallized from suitable solvents.

\section{General Procedure for Synthesis of Compounds 2 (a-i)}

The product obtained in step 1, compound 1 (10 mmol), phosphorous pentachloride $(10 \mathrm{mmol})$ and phosphorous oxychloride $(20 \mathrm{ml})$ was heated in a water bath for 4 to 4.5 hours and the reaction mixture was poured in crushed ice. The product obtained was filtered off, then dried and recrystallized from a suitable solvent.

\section{General Procedure for Synthesis of Compounds 3 (a-d)}

A mixture of compound $2(5 \mathrm{mmol})$ and hydrazine hydrate $(5 \mathrm{mmol})$ was taken in $20 \mathrm{ml}$ methanol and stirred for nine hours. The product obtained was filtered, dried and recrystallized from a suitable solvent.

\section{General Procedure for Synthesis of Compounds 4 (a-b)}

A mixture of compound $3(5 \mathrm{mmol})$, and an aldehyde $(5 \mathrm{mmol})$ was taken in $20 \mathrm{ml}$ methanol and refluxed for four hours. After that, the reaction mixture was poured in crushed ice. The product obtained was filtered, dried and recrystallized from a suitable solvent.

\section{Spectral Data of Selected Compounds}

Compound 1a: Yield 68\%, m. p. 271-272 ${ }^{\circ} \mathrm{C}$; IR (KBr) $\left(\mathrm{v} \mathrm{cm}^{-1}\right)$; $1712(\mathrm{C}=\mathrm{O}), 2230(\mathrm{CN}), 3301(\mathrm{NH})$, 1370- 1024 ( C-N Bending), 1458, 1590 ( $\mathrm{C}=\mathrm{C}$, Ar), 1610 (C=C) ; 1H NMR ( $300 \mathrm{MHz}$ ) ( $\delta$, ppm);7.017.28 (m,5H, Ar-H), 7.46( s,1H, Sec NH), 12.13 (s, 1H, amine).

Compound 1b: : Yield 84\%, m. p. 55-57 ${ }^{\circ} \mathrm{C}$; IR(KBr) $\left(\mathrm{v} \mathrm{cm}^{-1}\right)$; $1716(\mathrm{C}=\mathrm{O}), 2233(\mathrm{CN}), 3305(\mathrm{NH})$, 1371- 1021 ( C-N Bending), 1462, 1600 ( $\mathrm{C}=\mathrm{C}, \mathrm{Ar}), 1602(\mathrm{C}=\mathrm{C})$; $1 \mathrm{H}$ NMR ( $300 \mathrm{MHz})$ ( $\delta, \mathrm{ppm}) ; 3.78$ $\left(3 \mathrm{H}, \mathrm{s}, \mathrm{OCH}_{3}\right), 7.05-7.31(\mathrm{~m}, 4 \mathrm{H}, \mathrm{Ar}-\mathrm{H}), 7.51(\mathrm{~s}, 1 \mathrm{H}, \mathrm{Sec} \mathrm{NH}), 12.09$ (s, 1H, amine).

Compound 1c : Yield 72\%, m. p. 60-62 ${ }^{\circ} \mathrm{C}$; IR( $\left.\mathrm{KBr}\right)\left(\mathrm{v} \mathrm{cm}^{-1}\right) ; 1693(\mathrm{C}=\mathrm{O}), 2240(\mathrm{CN}), 3286(\mathrm{NH}), 1261$ ( C-N Bending), 1438 ( C=C), $3181(\mathrm{C}-\mathrm{H}), 3500(\mathrm{OH}) ; 1 \mathrm{H}$ NMR ( $300 \mathrm{MHz})(\delta, \mathrm{ppm}) ; 7.05-7.31(\mathrm{~m}, 4 \mathrm{H}$, Ar-H), 7.51( s,1H, Sec NH), $10.12(1 \mathrm{H}, \mathrm{s}, \mathrm{OH}), 12.09$ (s, 1H, amine).

Compound 1d: Yield 85\%, m. p. 74-76 ${ }^{\circ}$; IR $(\mathrm{KBr})\left(\mathrm{v} \mathrm{cm}^{-1}\right) ; 840(\mathrm{C}-\mathrm{Cl}), 1682(\mathrm{C}=\mathrm{O}), 2232(\mathrm{CN}), 3268$ (NH), 1246 (C-N Bending), 1445 ( $\mathrm{C}=\mathrm{C}), 3176(\mathrm{C}-\mathrm{H})$.

Compound 1e: Yield 72\%, m. p. 68-70 ${ }^{\circ} \mathrm{C}$; $1 \mathrm{H}$ NMR (300 MHz) ( $\delta$, ppm); 7.16-7.37 (m, 4H, Ar H), 7.55 (s, 1H, Sec NH), 12.19 (s, 1H, NH amine).

Compound 1f: Yield 90\%, m. p. 64-66 ${ }^{\circ}$; $1 \mathrm{H} \mathrm{NMR} \mathrm{(300} \mathrm{MHz)} \mathrm{( \delta ,} \mathrm{ppm);} \mathrm{7.21-7.46} \mathrm{(m,} \mathrm{4H,} \mathrm{Ar} \mathrm{H),} 7.55$ (s, 1H, Sec NH), 12.24 ( s, 1H, NH amine). 
RASĀYAN $J$. Chem.

Vol. 11 | No. 3 |1217 - 1221 | July - September | 2018<smiles>[R]c1ccc(-c2[nH]c(=S)[nH]c(=O)c2C#N)cc1</smiles><smiles>[R]c1ccc(C=Nc2nc(=S)[nH]c(-c3ccccc3)c2C#N)cc1</smiles>

$\begin{array}{ll}1 \mathrm{a}, \mathrm{R}=\mathrm{H} & 2 \mathrm{a}, \mathrm{R}=\mathrm{H} \\ 1 \mathrm{~b}, \mathrm{R}=p-\mathrm{OMe} & 2 \mathrm{~b}, \mathrm{R}=p-\mathrm{OMe} \\ 1 \mathrm{c}, \mathrm{R}=3-\mathrm{OH} & 2 \mathrm{c}, \mathrm{R}=3-\mathrm{OH} \\ 1 \mathrm{~d}, \mathrm{R}=3-\mathrm{Cl} & 2 \mathrm{~d}, \mathrm{R}=3-\mathrm{Cl} \\ 1 \mathrm{e}, \mathrm{R}=4-\mathrm{Cl} & 2 \mathrm{e}, \mathrm{R}=4-\mathrm{Cl} \\ 1 \mathrm{f}, \mathrm{R}=4-\mathrm{NO}_{2} & 2 \mathrm{f}, \mathrm{R}=4-\mathrm{NO}_{2} \\ 1 \mathrm{~g}, \mathrm{R}=2-\mathrm{Cl} & 2 \mathrm{~g}, \mathrm{R}=2-\mathrm{Cl} \\ 1 \mathrm{~h}, \mathrm{R}=3-\mathrm{NO}_{2} & 2 \mathrm{~h}, \mathrm{R}=3-\mathrm{NO}_{2} \\ 1 \mathrm{i}, \mathrm{R}=4-\mathrm{OH} & 2 \mathrm{i}, \mathrm{R}=4-\mathrm{OH}\end{array}$<smiles>[R]c1ccc(-c2[nH]c(=S)nc(Cl)c2C#N)cc1</smiles>

3(a-d)

Scheme-1

$$
\text { 3a, } \mathrm{R}=3-\mathrm{Cl} \quad 4 \mathrm{a}, \mathrm{R}=4-\mathrm{NO}_{2}, \mathrm{R} "=4-\mathrm{Cl}
$$

$3 \mathrm{~b}, \mathrm{R}=4-\mathrm{NO}_{2}$

$4 \mathrm{~b}, \mathrm{R}=4-\mathrm{NO}_{2}, \mathrm{R} "=3-\mathrm{OH}$

$3 \mathrm{c}, \mathrm{R}=2-\mathrm{OH}$

$3 \mathrm{~d}, \mathrm{R}=4-\mathrm{OH}$

2(a-i)

Compound 1g: Yield 80\%, m. p. 55-57 ${ }^{\circ} \mathrm{C}$; IR (KBr) $\left(v \mathrm{~cm}^{-1}\right) ; 848(\mathrm{C}-\mathrm{Cl}), 1673(\mathrm{C}=\mathrm{O}), 2253(\mathrm{CN}), 3257$ (NH), 1242 (C-N Bending), $1438(\mathrm{C}=\mathrm{C}), 3168(\mathrm{C}-\mathrm{H})$.

Compound 1h: Yield 78\%, m. p. 130-132 ${ }^{\circ} \mathrm{C} ; 1 \mathrm{H} \mathrm{NMR}(300 \mathrm{MHz})(\delta, \mathrm{ppm}) ; 7.24-7.51(\mathrm{~m}, 4 \mathrm{H}, \mathrm{Ar} \mathrm{H})$, 7.46 (s, 1H, Sec NH), 12.16 (s, 1H, NH amine).

Compound 1i: Yield 80\%, m. p. 168-170 ${ }^{\circ} \mathrm{C}$; $\mathrm{IR}(\mathrm{KBr})\left(\mathrm{v} \mathrm{cm} \mathrm{cm}^{-1}\right) ; 1688(\mathrm{C}=\mathrm{O}), 2234(\mathrm{CN}), 3277(\mathrm{NH})$, 1252 ( C-N Bending), $3176(\mathrm{C}-\mathrm{H}), 1431(\mathrm{C}=\mathrm{C}), 3512(\mathrm{OH}) ; 1 \mathrm{H}$ NMR ( $300 \mathrm{MHz})(\delta$, ppm); 7.12-7.33 (m, 4H, Ar H), 7.48 ( s, 1H, Sec NH), $10.10(1 \mathrm{H}, \mathrm{s}, \mathrm{OH}) 12.11$ (s, 1H, amine).

Table-1: Physical and Analytical Data of the Prepared Compounds (1a-i), (2a-i), (3a-d) and (4a-b)

\begin{tabular}{c|c|c|c|c|c}
\hline S. No. & Compound Code & Molecular Formula & Molecular Wt. & Melting Point $\left({ }^{\circ} \mathrm{C}\right)$ & $\begin{array}{c}\text { Yield } \\
\%\end{array}$ \\
\hline 1 & $1 \mathrm{a}$ & $\mathrm{C}_{11} \mathrm{H}_{7} \mathrm{~N}_{3} \mathrm{SO}$ & 229.0 & $271-272$ & 68 \\
\hline 2 & $1 \mathrm{~b}$ & $\mathrm{C}_{12} \mathrm{H}_{9} \mathrm{~N}_{3} \mathrm{O}_{2} \mathrm{~S}$ & 259.0 & $55-57$ & 84 \\
\hline 3 & $1 \mathrm{c}$ & $\mathrm{C}_{11} \mathrm{H}_{7} \mathrm{~N}_{3} \mathrm{O}_{2} \mathrm{~S}$ & 245.0 & $60-62$ & 72 \\
\hline 4 & $1 \mathrm{~d}$ & $\mathrm{C}_{11} \mathrm{H}_{6} \mathrm{~N}_{3} \mathrm{SOCl}$ & 263.5 & $74-76$ & 85 \\
\hline 5 & $1 \mathrm{e}$ & $\mathrm{C}_{11} \mathrm{H}_{6} \mathrm{~N}_{3} \mathrm{SOCl}$ & 263.5 & $68-70$ & 72 \\
\hline 6 & $1 \mathrm{f}$ & $\mathrm{C}_{11} \mathrm{H}_{6} \mathrm{~N}_{4} \mathrm{O}_{3} \mathrm{~S}$ & 274.0 & $64-66$ & 90 \\
\hline 7 & $1 \mathrm{~g}$ & $\mathrm{C}_{11} \mathrm{H}_{6} \mathrm{~N}_{3} \mathrm{SOCl}$ & 263.5 & $55-57$ & 80 \\
\hline 8 & $1 \mathrm{~h}$ & $\mathrm{C}_{11} \mathrm{H}_{6} \mathrm{~N}_{4} \mathrm{O}_{3} \mathrm{~S}$ & 274.0 & $130-132$ & 78 \\
\hline 9 & $1 \mathrm{i}$ & $\mathrm{C}_{11} \mathrm{H}_{7} \mathrm{~N}_{3} \mathrm{O}_{2} \mathrm{~S}$ & 245.0 & $168-170$ & 80 \\
\hline 10 & $2 \mathrm{a}$ & $\mathrm{C}_{11} \mathrm{H}_{6} \mathrm{~N}_{3} \mathrm{SCl}$ & 246.5 & $186-188$ & 73 \\
\hline 11 & $2 \mathrm{~b}$ & $\mathrm{C}_{12} \mathrm{H}_{8} \mathrm{~N}_{3} \mathrm{SOCl}$ & 277.5 & $178-180$ & 88 \\
\hline & \multicolumn{2}{|r}{} & &
\end{tabular}


RASĀYAN J. Chem.

Vol. 11 | No. 3 |1217 - 1221 | July - September | 2018

\begin{tabular}{c|c|c|c|c|c}
\hline 12 & $2 \mathrm{c}$ & $\mathrm{C}_{11} \mathrm{H}_{6} \mathrm{~N}_{3} \mathrm{SOCl}$ & 263.5 & $212-214$ & 70 \\
\hline 13 & $2 \mathrm{~d}$ & $\mathrm{C}_{11} \mathrm{H}_{5} \mathrm{~N}_{3} \mathrm{SCl}_{2}$ & 282.0 & $160-162$ & 81 \\
\hline 14 & $2 \mathrm{e}$ & $\mathrm{C}_{11} \mathrm{H}_{5} \mathrm{~N}_{3} \mathrm{SCl}_{2}$ & 282.0 & $160-162$ & 76 \\
\hline 15 & $2 \mathrm{f}$ & $\mathrm{C}_{11} \mathrm{H}_{5} \mathrm{~N}_{4} \mathrm{O}_{2} \mathrm{SCl}$ & 292.5 & $208-210$ & 80 \\
\hline 16 & $2 \mathrm{~g}$ & $\mathrm{C}_{11} \mathrm{H}_{5} \mathrm{~N}_{3} \mathrm{SCl} \mathrm{Cl}_{2}$ & 282.0 & $158-160$ & 84 \\
\hline 17 & $2 \mathrm{~h}$ & $\mathrm{C}_{11} \mathrm{H}_{5} \mathrm{~N}_{4} \mathrm{O}_{2} \mathrm{SCl}$ & 292.5 & $158-160$ & 77 \\
\hline 18 & $2 \mathrm{i}$ & $\mathrm{C}_{11} \mathrm{H}_{6} \mathrm{~N}_{3} \mathrm{SOCl}$ & 263.5 & $218-220$ & 73 \\
\hline 19 & $3 \mathrm{a}$ & $\mathrm{C}_{11} \mathrm{H}_{8} \mathrm{~N}_{5} \mathrm{SCl}$ & 277.5 & $212-214$ & 65 \\
\hline 20 & $3 \mathrm{~b}$ & $\mathrm{C}_{11} \mathrm{H}_{8} \mathrm{~N}_{6} \mathrm{SO} \mathrm{O}_{2} \mathrm{Cl}$ & 288.0 & $181-183$ & 68 \\
\hline 21 & $3 \mathrm{c}$ & $\mathrm{C}_{11} \mathrm{H}_{9} \mathrm{~N}_{5} \mathrm{SOCl}$ & 259.0 & $191-193$ & 60 \\
\hline 22 & $3 \mathrm{~d}$ & $\mathrm{C}_{11} \mathrm{H}_{9} \mathrm{~N}_{5} \mathrm{SOCl}$ & 259.0 & $224-226$ & 62 \\
\hline 23 & $4 \mathrm{a}$ & $\mathrm{C}_{18} \mathrm{H}_{11} \mathrm{~N}_{6} \mathrm{O}_{2} \mathrm{SCl}$ & 410.5 & $188-190$ & 66 \\
\hline 24 & $4 \mathrm{~b}$ & $\mathrm{C}_{18} \mathrm{H}_{12} \mathrm{~N}_{6} \mathrm{O}_{3} \mathrm{~S}$ & 392.0 & $157-159$ & 61 \\
\hline
\end{tabular}

Compound 2a: Yield 73\%, m. p.186-188 ${ }^{\circ} \mathrm{C}$; IR $(\mathrm{KBr})\left(\mathrm{v} \mathrm{cm}^{-1}\right)$; 1476, 1618 ( C=C, Ar) 1617 ( C=C), $1678(\mathrm{C}=\mathrm{N}), 2230(\mathrm{CN})$, and $3316(\mathrm{Sec} \mathrm{NH}) ; 1 \mathrm{H} \mathrm{NMR}(300 \mathrm{MHz})(\delta, \mathrm{ppm}) ; 7.2-7.4(\mathrm{~m}, 5 \mathrm{H}, \mathrm{Ar}-\mathrm{H}), 8.7$ $(\mathrm{s}, 1 \mathrm{H}, \mathrm{NH})$.

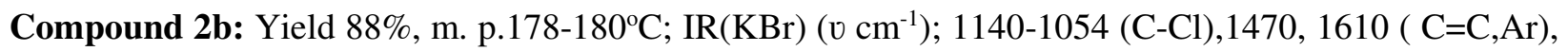
$1615(\mathrm{C}=\mathrm{C}), 1681(\mathrm{C}=\mathrm{N}), 2227(\mathrm{CN})$, and $3310(\mathrm{Sec} \mathrm{NH}) ; 1 \mathrm{H} \mathrm{NMR}(300 \mathrm{MHz})(\delta, \mathrm{ppm}) ; 3.76(3 \mathrm{H}, \mathrm{s}$, $\left.\mathrm{OCH}_{3}\right), 7.2-8.4(\mathrm{~m}, 4 \mathrm{H}, \mathrm{Ar}-\mathrm{H}), 8.7(\mathrm{~s}, 1 \mathrm{H}, \mathrm{NH})$.

Compound 2c: Yield 70\%, m. p.212-214 ${ }^{\circ} \mathrm{C}$; IR (KBr) $\left(\mathrm{v} \mathrm{cm}^{-1}\right) ; 1445-1048$ ( C-Cl), 1462, 1618 ( C=C, Ar) 1608( C=C), $1672(\mathrm{C}=\mathrm{N}), 2232(\mathrm{CN})$, and $3306(\mathrm{Sec} \mathrm{NH}), 3512(\mathrm{OH}) ; 1 \mathrm{H} \mathrm{NMR}(300 \mathrm{MHz})(\delta$, ppm); 7.1-7.5 (m,5H, Ar-H), 8.6(s,1H, NH), 10.01( 1H, s, OH).

Compound 2d: Yield 81\%, m. p.160-162 ${ }^{\circ} \mathrm{C}$; $1 \mathrm{H}$ NMR (300 MHz) ( $\left.\delta, \mathrm{ppm}\right)$; 7.3-7.3 (m,4H, Ar-H), 8.6( $\mathrm{s}, 1 \mathrm{H}, \mathrm{NH})$.

Compound 2e: Yield 76\%, m. p.160-162 $\mathrm{C}$; IR (KBr) (v cm $\left.{ }^{-1}\right)$; 1138-1041 (C-Cl), 1465, 1622 ( C=C,Ar) $1612(\mathrm{C}=\mathrm{C}), 1678(\mathrm{C}=\mathrm{N}), 2224(\mathrm{CN})$, and $3314(\mathrm{Sec} \mathrm{NH})$.

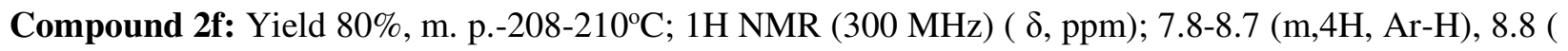
$\mathrm{s}, 1 \mathrm{H}, \mathrm{NH})$.

Compound 2g: Yield 84\%, m.p.158-160 ${ }^{\circ}$; $1 \mathrm{H}$ NMR (300 MHz) ( $\left.\delta, \mathrm{ppm}\right) ; 7.9-8.8(\mathrm{~m}, 4 \mathrm{H}, \mathrm{Ar}-\mathrm{H}), 8.6($ $\mathrm{s}, 1 \mathrm{H}, \mathrm{NH})$.

Compound 2h: Yield 77\%, m. p.158-160'C; IR (KBr) (v cm $\left.{ }^{-1}\right)$; 1120-1042 (C-Cl), 1455, 1613 (C=C, Ar) $1622(\mathrm{C}=\mathrm{C}), 1550\left(\mathrm{NO}_{2}\right), 1663(\mathrm{C}=\mathrm{N}), 2235(\mathrm{CN})$, and $3327(\mathrm{Sec} \mathrm{NH})$.

Compound 2i: Yield 73\%, m. p. 218-220 ${ }^{\circ} \mathrm{C}$; $1 \mathrm{H} \mathrm{NMR} \mathrm{(300} \mathrm{MHz)} \mathrm{(} \delta$, ppm); 7.7-8.6 (m,4H, Ar-H), 8.4( $\mathrm{s}, 1 \mathrm{H}, \mathrm{NH}), 10.12(1 \mathrm{H}, \mathrm{S}, \mathrm{OH})$.

Compound 3a: Yield 65\%, m. p.212-214 ${ }^{\circ} \mathrm{C}$; IR(KBr) $\left(\mathrm{v} \mathrm{cm}^{-1}\right) ; 770(-\mathrm{Cl}), 1405-1020$ ( C-N), 1460, 1620 ( $\mathrm{C}=\mathrm{C}, \mathrm{Ar}) 1618(\mathrm{C}=\mathrm{C}), 2215(\mathrm{CN}), 3130(\mathrm{Sec} \mathrm{NH}), 3410\left(\operatorname{pri}_{\mathrm{NH}}\right)$; 1H NMR ( $\left.300 \mathrm{MHz}\right)(\delta, \mathrm{ppm})$; 7.30-8.30 (m,4H, Ar-H), 8.60 ( s,2H, NH), 8.40 ( s, 2H, - $\mathrm{NH}_{2}$ ).

Compound 3b: Yield 68\%, m. p.181-183 ${ }^{\circ} \mathrm{C}$; $\mathrm{IR}(\mathrm{KBr})\left(\mathrm{v} \mathrm{cm}^{-1}\right)$; 1403-1018 ( C-N), 1458, $1615(\mathrm{C}=\mathrm{C}$, Ar) $1620(\mathrm{C}=\mathrm{C}), 2220(\mathrm{CN}), 3126(\mathrm{Sec} \mathrm{NH}), 3412\left(\operatorname{pri}_{\mathrm{NH}}\right), 1550\left(-\mathrm{NO}_{2}\right) ; 1 \mathrm{H} \mathrm{NMR}(300 \mathrm{MHz})(\delta$, ppm); 7.32-8.38 (m,4H, Ar-H), 8.46 ( s,2H, NH), 8.23 ( s, 2H, - $\left.\mathrm{NH}_{2}\right)$.

Compound 3c: Yield 60\%, m. p. 191-193 ${ }^{\circ} \mathrm{C}$; IR(KBr) $\left(\mathrm{v} \mathrm{cm}^{-1}\right)$; 1406-1022 ( C-N), 1462, 1622 ( C=C, Ar), 1620 ( C=C), 2218( CN), 3133 (Sec NH), 3401 ( pri. NH), 3320 ( -OH ).

Compound 3d: Yield 62\%, m .p.224-226 ${ }^{\circ}$; IR(KBr) $\left(\mathrm{v} \mathrm{cm}^{-1}\right)$; 1407-1023 ( C-N), 1456, 1624 ( C=C, Ar) 1621 ( C=C), 2220( CN), 3126 (Sec NH), 3408 (pri. NH), $3316(-\mathrm{OH})$. 
Compound 4a: Yield 66\%, m. p.188-190 $\mathrm{C}$; IR(KBr) $\left(\mathrm{v} \mathrm{cm}^{-1}\right)$; $778(-\mathrm{Cl}), 1408-1022$ ( C-N), 1462, 1618 ( $\mathrm{C}=\mathrm{C}$, Ar) $1620(\mathrm{C}=\mathrm{C}), 2216(\mathrm{CN}), 3135(\mathrm{Sec} \mathrm{NH}), 1552\left(-\mathrm{NO}_{2}\right)$; 1H NMR ( $\left.300 \mathrm{MHz}\right)(\delta, \mathrm{ppm})$; 7.22-8.40 (m,8H, Ar-H), 9.60 ( s,2H, NH), 9.13 ( s, 1H, -CH).

Compound 4b: Yield 61\%, m. p.157-159 C; IR (KBr) $\left(\mathrm{v} \mathrm{cm}^{-1}\right)$; 1410-1020 ( C-N), 1460, 1624 ( C=C, Ar) $1621(\mathrm{C}=\mathrm{C}), 2220(\mathrm{CN}), 3126(\mathrm{Sec} \mathrm{NH}), 3316(-\mathrm{OH}), 1554\left(-\mathrm{NO}_{2}\right)$; $1 \mathrm{H} \mathrm{NMR}(300 \mathrm{MHz})(\delta$, ppm); 7.20-8.38 (m,8H, Ar-H), 9.80 ( $\mathrm{s}, 1 \mathrm{H}, \mathrm{OH}), 9.18$ ( s, 1H, -CH).

\section{RESULTS AND DISCUSSION}

The development of efficient and environmentally acceptable synthetic methods is an important task of modern chemistry. Hence we have developed a synthetic protocol for the synthesis of some new biologically active pyrimidine compounds. Pyrimidine compounds have occupied an important position in natural and synthetic organic chemistry, mainly due to their wide range of biological activities.

\section{CONCLUSION}

In the present investigation, some new pyrimidine compounds have been synthesized in moderate to good yields. It is observed that yields are better with electron withdrawing groups as compared with electron releasing groups in aldehydes.

\section{ACKNOWLEDGMENT}

The author is thankful to University Grants Commission, New Delhi for providing financial assistance.

\section{REFERENCES}

1. C. D. Dave, R. D. Shah, Molecules, 7, 554(2002), DOI:10.3390/70700554

2. A. Gangiee, O. Adaira, S.F. Queener, Bioorg. Med. Chem., 9, 2929(2001), DOI: 10.1016/S09680896(01)00223-1

3. R. R. Patel, V.G. Patel, RASAYAN Journal of Chemistry, 3(1), 188(2010)

4. G.R. Madhavan, R. Chakrabarti, R.K. Vikramadithyan, R.N. Mamidi, V.S. Balraju, B.M. Rajesh, P.Misra, S.K.B. Kumar, B.B. Lohray, V.B. Lohray, Bioorg. Med. Chem., 10, 2671(2002), DOI: 10.1016/S0968-0896(02)00107-4

5. S.I. Pretorius, W.J. Breytenbach, C. de Kock, P.J. Smith, D.D.N'da, Bioorg. Med. Chem., 21, 269 (2013), DOI: 10.1016/j.bmc.2012.10.019

6. P. Shanmugasundaram, J. Mohanarangan, R.K. Raj, M.V. Anandhi, RASAYAN Journal of Chemistry, 2(2), 345(2009).

7. E.S. Zuniga, A. Korkegian, S. Mullen, E.J. Hembre, P.L. Ornstein, G. Cortez, K. Biswas, K. Naresh, J. Cramer, T. Masqulin, Bioorg. Med. Chem., 25, 3922(2017), DOI: 10.1016/j.bmc.2017.05.030.

8. A. Kumar, S Sinha, P.M. Chauhan.Bioorg.Med. Chem. Lett., 12, 667(2012), DOI:10.1016/S0960894X(01)00829-0

9. S.M. Sondhi, M. Johar, S. Rajvanshi, Aust. J. Chem., 54, 69(2001), DOI: 10.1071/CH00141

10. A. Gangjee, A Vidwans, E Elzein, J. J. McGuire, S.F. Queener, R.L. Kisliuk, J. Med. Chem., 44, 1993(2001), DOI: 10.1021/jm0100382

11. Md. Salahuddin, S. Singh, S.M. Shantakumar, RASAYAN Journal of Chemistry, 2(1), 167(2009).

12. P.G. Baraldi, M.G. Pavani, M. Nunez, Bioorg. Med. Chem., 10, 449(2002), DOI:10.1016/S09680896(01)00294-2

13. M. N. Nasr, M. M. Gineinah, Arch Pharm., 335, 289(2002), DOI: 10.1002/1521-4184(200208)335

14. A. Corma, V. Fornes, R.M. Martin- Aranda, H. Garcia, J. Appl. Catal., 59, 237(1990), DOI: 10.1016/S0166-9834(00)82201-0

15. B. Veldurthy., J. M. Clacens, F. Figueras, Adv. Synth. Catal., 347, 767(2005), DOI: 10.1002/adsc.200404371

16. C. Xu, J. K. Bartley, D. I. Enache, D.W. Knight, G. J. Hutchings, Synthesis, 10, 3468(2005), DOI: 10.1055/s-2005-918467

[RJC-3062/2018] 